\title{
Actitudes lingüísticas, revisión teórica y repercusiones en la enseñanza de lenguas extranjeras*
}

MÓNICA CHAMORRO MEJÍA**

Recepción: 13 de octubre de 2020

Aprobación: 8 de enero de 2021

Forma de citar este artículo: Chamorro, M. (2021). Actitudes lingüísticas, revisión teórica y repercusiones en la enseñanza de lenguas extranjeras. Cuadernos de Lingüística Hispánica, (37), e11841.

(30) https://10.19053/0121053X.n37.2021.11841

* Artículo de revisión

** Doctora en Lingüística Aplicada de la Universidad de Alcalá de Henares (España). Docente de la Universidad del Valle, (Colombia). Correo electrónico: monica.chamorro@correounivalle.edu.co. https://orcid.org/ 0000$\underline{0003-4953-4591}$ 


\section{Resumen}

El objetivo de este artículo es realizar una revisión general del campo de las actitudes lingüísticas y, en particular, de sus aplicaciones en la enseñanza de las lenguas extranjeras. Se inicia con una ubicación disciplinar de las actitudes lingüísticas, tomando en cuenta los principales autores y líneas teóricas. Asimismo, se indaga acerca de sus relaciones con la conciencia lingüística, la inseguridad lingüística, los estereotipos y los prejuicios. Después, se examina el problema de la definición de las actitudes lingüísticas y la caracterización de sus diversos componentes desde diferentes enfoques. De igual forma, se analizan las relaciones entre estilo, prestigio y estudios de actitudes lingüísticas en el ámbito educativo y la manera en que influye la formación de las actitudes lingüísticas en los procesos de enseñanza/aprendizaje de lenguas extranjeras. Finalmente, se estudian las conexiones entre actitudes lingüísticas, motivación, adquisición de lenguas y la forma en la que intervienen estas conexiones en la competencia comunicativa y en los procesos de acomodación comunicativa del hablante de una lengua.

Palabras clave: sociolingüística, psicolingüística, investigación lingüística, etnolingüística.

\section{Linguistic Attitudes, Theoretical Review and Repercussions on Foreign Language Teaching}

\section{Abstract}

The aim of this paper is to carry out a general review of the field of linguistic attitudes and, of its application in foreign languages teaching. It begins with a disciplinary placement of linguistic attitudes, taking into account the main authors and theoretical lines. Likewise, it inquires about their relationships with linguistic awareness, linguistic insecurity, stereotypes and prejudices. Then, the problem of defining linguistic attitudes and the characterization of their various components from different approaches is examined. Similarly, relationships between style, prestige and linguistic attitudes studies in the educational field are analyzed, and how formation of linguistic attitudes influences the teaching/learning processes of foreign languages. Finally, connections between linguistic attitudes, motivation, language acquisition and how these connections intervene in communicative competence and in the processes of communicative accommodation of the language speaker are studied.

Keywords: sociolinguistics, psycholinguistics, linguistic research, ethnolinguistics 


\section{Attitudes linguistiques, révision théorique et retombées sur l'enseignement des langues étrangères}

\section{Résumé}

Cet article tourne autour d'une révision générale du champ des attitudes linguistiques et, notamment, de leurs applications dans le domaine de l'enseignement des langues étrangères. D'abord, un locus disciplinaire des attitudes linguistiques est effectué, tout en tenant compte des principaux auteurs et de ses lignes théoriques. On prétend, également, établir leurs rapports avec la conscience et l'insécurité linguistiques, les stéréotypes et les préjugés. Par ailleurs, la révision se focalise sur le problème de la définition des attitudes linguistiques et de leur caractérisation à la lumière des distinctes études et approches, de leurs différentes composantes. De la même manière, on analyse les rapports entre le style, le statut et les études des attitudes linguistiques dans le domaine éducatif et la façon dont leur formation détermine les processus d'enseignement / apprentissage des langues étrangères. Finalement, on étudie les liens entre les attitudes linguistiques, la motivation, l'acquisition de la langue et la manière dont ces connexions interviennent dans la compétence communicative et dans les processus d'assimilation communicative du locuteur d'une langue.

Mots clés: sociolinguistique, psycholinguistique, recherche linguistique, ethnolinguistique.Atitudes em línguas, revisão teórica e repercussões no ensino de línguas estrangeiras

\section{Atitudes em línguas, revisão teórica e repercussões no ensino de línguas estrangeiras}

\section{Resumo}

Este artigo se concentra em uma revisão geral do campo das atitudes linguísticas e, em particular, suas aplicações no campo do ensino de línguas estrangeiras. Em princípio, é realizada uma localização disciplinar das atitudes linguísticas, levando em consideração os principais autores e linhas teóricas. Da mesma forma, indaga sobre suas relações com a consciência linguística, a insegurança linguística, os estereótipos e os preconceitos. A seguir, a revisão enfoca o problema da definição das atitudes linguísticas e sua caracterização a partir das diferentes abordagens, de seus vários componentes. Da mesma forma, são analisadas as relações entre estilo, prestígio e estudos de atitudes linguísticas no campo educacional e a forma como a formação de atitudes linguísticas influencia os processos de ensino / aprendizagem de línguas estrangeiras. Por fim, são estudadas as conexões entre as atitudes linguísticas, a motivação, a aquisição da linguagem e a forma como essas conexões interferem na competência comunicativa e nos processos de acomodação comunicativa do falante de uma língua.

Palavras-chave: sociolinguística, psicolinguística, pesquisa linguística, etnolinguístic 


\section{Introducción}

En este artículo nos proponemos hacer una revisión general del campo de las actitudes lingüísticas, rastreando sus orígenes en la psicología y la psicología social, sus desarrollos concretos en la sociolingüística (espacio disciplinar desde el que se definen y caracterizan) y, en último término, ofrecer una perspectiva de sus relaciones con la didáctica de las lenguas, en particular en lo concerniente a los resultados de los procesos de adquisición, la motivación y el diseño didáctico.

El estudio de las actitudes lingüísticas (en adelante AL) pertenece al campo de conocimiento de la sociolingüística y por ende, según la definición de Hudson (1980), al estudio del lenguaje con relación a la sociedad. Sin embargo, con respecto a la pertenencia disciplinar esta afirmación no está exenta de controversias, dado que algunos aspectos teóricos y aproximaciones metodológicas desbordan el ámbito de la sociolingüística variacionista. Por perspectiva variacionista se entiende, según García (1999), aquella que Labov (1972) denomina sociolingüística estricta, y López (1989), sociolingüística hacia adentro. Se trata de aquella que busca centrarse en la forma en que la sociedad incide en la variación de los niveles de una lengua (y no al contrario, como la denominada sociolingüística amplia o sociolingüística hacia afuera). De hecho, las AL se desplazan hacia el campo de la sociología del lenguaje, de la etnografía del habla o de la didáctica de las lenguas, en la medida en que indagan sobre los aspectos psicológicos que no necesariamente se expresan en variaciones de tipo lingüístico, sino que se refieren, por ejemplo, a la selección de un estilo de acuerdo con los contextos comunicativos o a las dificultades de aprendizaje escolar de una lengua dada. Desde este punto de vista, García (1999) describe la complejidad disciplinar de las AL, señalando que tal complejidad es inherente a la "trabada red de valores" (p. 145) que incide en la relación entre sociedad y lengua.

\section{Conciencia lingüística, inseguridad lingüística y actitudes lingüísticas}

Los hablantes establecen una red de valores a partir de la cual determinan sus percepciones acerca de la lengua (bien sea la propia o la ajena), de las variedades de la misma lengua o de lenguas diferentes. Esta red de valores hace referencia al concepto de conciencia lingüística que, para García (1999), es el "saber que los hablantes tienen, tanto de la estratificación social de las variantes, variedades y/o lenguas que pueden integrar el repertorio verbal de una comunidad, como de los parámetros valorativos que se les adjudican" (pp. 147-148). En este sentido, la conciencia lingüística depende no solo de que el hablante disponga de recursos lingüísticos equifuncionales, sino de que además disponga de una variación social de los mismos. La conciencia lingüística genera un valor de seguridad 0 inseguridad en el hablante y está determinada por el modelo de lengua del hablante. Este modelo es un espacio singular, resultado de la forma en la que el individuo filtra sus experiencias y comprende parámetros lingüísticos y extralingüísticos (Hudson, 1980). Así, la conciencia lingüística no es un fenómeno de grupo, sino un fenómeno individual que acontece en un ambiente social 
dado. Conceptos estrechamente ligados a la formación de la conciencia lingüística, tales como la inseguridad/seguridad, el modelo de lengua, los estereotipos y prejuicios, desempeñan un rol decisivo en la caracterización de las AL.

Sin embargo, la conciencia lingüística no abarca todos los aspectos de las AL, como se verá a continuación; hay otros factores relacionados con el comportamiento y con la interacción comunicativa que las involucran, moldean y transforman. Con relación a la edad de formación de las AL, los resultados de las investigaciones son contrastantes. Los estudios de actitudes lingüísticas (en adelante EAL) de Lambert (1967) realizados en niños bilingües (inglés/francés), arrojaron datos que situaron la edad de conciencia del propio idiolecto en torno a los doce años. Posteriormente, un estudio de Giles y Powesland (1975) en niños británicos demostró que estos adquieren una valoración acerca de los sociolectos hacia los doce años de edad y solo hasta los diecisiete años acerca de la propia habla. Por último, los estudios de Schneiderman (1976) en el ámbito canadiense disminuyeron el rango de edad señalado a los cinco años.

\section{Estereotipos, prejuicios y AL}

El prejuicio para Allport (1954) es una actitud hostil hacia un individuo que hace parte de un grupo social, a quien se le atribuyen características negativas de este colectivo. El prejuicio no es solamente un juicio racional de valor, sino que contiene un elemento emotivo o irracional que transfiere automáticamente las características del grupo al individuo y viceversa. El estereotipo permite fragmentar la realidad en un espectro de grupos sociales a los que se les adjudican determinadas categorías. Estas categorías tienden a homogeneizar a los individuos pertenecientes al mismo grupo y a acentuar las diferencias entre los grupos diversos; por lo tanto, el fenómeno de la homogeneización interna y de la diferenciación externa es la base del estereotipo.

En el campo de las AL, los estereotipos permiten que se establezcan predicciones y negociaciones en la interacción comunicativa entre las diferentes variedades 0 estilos de una lengua 0 , en el caso de las situaciones de lenguas en contacto, entre las diferentes lenguas. Esto es posible porque los estereotipos confinan a los hablantes dentro de los límites de grupos cerrados, que a su vez están incluidos en conjuntos más amplios, previamente definidos a partir de una red de valores que señalan fronteras de diversidad hacia el exterior de los grupos y de homogeneidad hacia el interior de estos.

Entre tanto, para Miquel (2004), desde la perspectiva concreta de la didáctica de la lengua, las presuposiciones, los símbolos, las creencias, los modos de clasificación y las actuaciones, son las unidades de análisis que nos permiten entender las diferentes culturas, influyen en las AL de los hablantes y, por ende, gobiernan las interacciones comunicativas. 


\section{Definición y características de las AL}

El interés multidisciplinar hacia los EAL ha implicado una aproximación múltiple a la cuestión y una heterogeneidad en su conceptualización y caracterización. Desde la psicología social, Fishbein y Ajzen (1975) definen las AL como “(..) the amount of affect for or against some object" (p. 11). En esta noción, el concepto de afecto (o desafecto) es central en la medida en que las experiencias afectivas del individuo son aquellas que lo predisponen a dar una respuesta consistente; es decir, mismo tipo de respuesta hacia un objeto (sujeto) dado. Allport (1954), por su parte, vincula el concepto de actitud con el de prejuicio, considerando que, junto con las creencias, determinan la actitud favorable o desfavorable. Las actitudes son mucho menos móviles que las creencias debido a que estas últimas pueden ser cambiadas a partir de una lógica o razonamiento. Esta distinción entre prejuicio, actitud y creencia resulta útil a medida que se enfatiza el aspecto intangible de las actitudes y permite establecer la dificultad de su observación directa. Otro autor como Oppenheim (1982), quien incorpora aspectos cognitivos y comportamentales a su caracterización, se inclina también por la intangibilidad de las AL, pues señala que solo pueden ser inferidas a partir de las reacciones emocionales de los sujetos. No obstante, estudios como el de La Piere (1934) demuestran que no es posible en todos los casos extrapolar la actitud del comportamiento, debido a que no siempre este último establece una relación coherente de causa/efecto con la actitud, lo que se manifiesta en que las personas en ocasiones no actúan de forma armónica con sus actitudes, sino que, por el contrario, el comportamiento puede controvertir la actitud.

Esta diversidad de miradas acerca de las AL ha dado lugar a caracterizaciones detalladas de los diferentes enfoques; entre los más importantes podemos mencionar el estudio clásico de Agheysi y Fishman (1970) y el más reciente de Garrett (2010), quien afirma que una actitud es una orientación evaluativa hacia un objeto social de algún tipo, sea este una lengua o una nueva política gubernamental, entre otros. Agheyisi y Fishman (1970) dividen la aproximación a las actitudes en dos enfoques: el mentalista y el conductista. Según Allport (1935), el enfoque mentalista supone que las actitudes son una predisposición mental y neuronal y, en consecuencia, son constructos hipotéticos que están confinados a un campo inferencial. Otros autores como Doob (1947), Chein (1948) y Green (1954), desde esta perspectiva mentalista han formulado una definición que si bien parte de la actitud como un constructo hipotético, tiene una relación directa con el comportamiento manifiesto o con respuestas verbales a estímulos dados. La perspectiva conductista, por su parte, pone el acento en la respuesta, es decir, en el comportamiento. Este enfoque es ventajoso en términos metodológicos, ya que al concebir las actitudes como un todo manifiesto no presenta problemas de observación y puede ser sometido a un tratamiento estadístico convencional. Sin embargo, la crítica a esta perspectiva se centra en la imposibilidad de independizar la actitud como una respuesta a un estímulo específico y, por lo tanto, siendo una variable dependiente (y no independiente como en el enfoque mentalista) no sirve para extrapolar o prever otro tipo de respuesta en condiciones similares a las observadas (Alexander \& Norman, 1967). Esta distinción entre enfoques mentalistas 
y conductistas ha sido tomada en cuenta por Fasold (1990) y en el ámbito hispánico por López (1989).

\section{Componentes de las AL}

Para Moreno (1998), las AL tienen tres elementos: una valoración (componente afectivo), un saber o creencia (componente cognoscitivo) y una conducta (componente conativo). Esta concepción tripartita, que prevé una complejidad innata de las $\mathrm{AL}$, es compartida por quienes se inclinan por un enfoque mentalista, como Lambert y Lambert (1964), Rosenberg (1960) y Rokeach (1968). Algunos autores que se enfocan en una visión conductista conciben las AL como unicomponenciales. Es el caso de Osgoog et al. (1957), quienes sostienen que las AL tienen un único componente afectivo. Por otra parte, para Fishbein (1965) existe una distinción entre actitud y creencia: la actitud tiene un solo componente, el afectivo, mientras que la creencia tiene dos, uno cognitivo y uno comportamental.

Otra forma de concebir las AL está más relacionada con la dinámica de la comunicación que con sus componentes. Esta teoría, la teoría de la acomodación comunicativa, proviene de la psicología social y hace referencia a la forma en la que los hablantes se adaptan en el acto comunicativo a las formas de interacción de sus respectivos partners. Giles (1973), quien formula originalmente la teoría, considera que existen estrategias de convergencia y de divergencia en la interacción. Las estrategias de convergencia buscan disminuir las diferencias entre los estilos del discurso de los participantes, y las de divergencia, buscan acentuar las diferencias. Estas estrategias pueden afectar diferentes niveles de la lengua (fonético/fonológico, léxico/semántico, morfosintáctico) o aspectos extralingüísticos de la comunicación. Cabe mencionar que tanto la convergencia como la divergencia están condicionadas por los estereotipos lingüísticos de una comunidad de habla. Asimismo, se puede observar que existe una convergencia asimétrica, según los hallazgos de White (1989). En este tipo de convergencia, uno de los participantes de la interacción puede usar estrategias de acomodación mientras que los demás participantes pueden no empeñarse en dichas estrategias.

Otro modelo de AL en el que prima la dinámica de la interacción sobre el enfoque o los componentes es el de Street y Hooper (1982). Este modelo sostiene que los procesos cognitivos y conativos se determinan a partir de los juicios de valor y de los usos lingüísticos. En la interacción comunicativa se parte de un proceso de acomodación del habla y a partir de la percepción del mensaje se manifiesta la respuesta valorativa. A su vez, esta respuesta está determinada por el ambiente de enunciación. Desde el campo netamente sociolingüístico, López (1989) propone un modelo en el que se distingue claramente entre actitud y creencia. Las actitudes dan origen a comportamientos (aspectos conativos) de aceptación y rechazo y son, desde este punto de vista, el único componente. Las creencias que dan origen a las actitudes están conformadas a su vez por aspectos relacionados con la cognición de los usos lingüísticos de una comunidad dada y por un componente afectivo ligado a los usos 
lingüísticos. Hay que resaltar que las actitudes pueden ser negativas o positivas, nunca neutras, pues estaríamos ante una ausencia de actitud.

\section{Estilo, estándar y AL}

Para Giles (1973, 2014), el estilo del discurso puede ser diagnosticado socialmente, estereotipado y dotado de un mayor o menor prestigio. Desde esta perspectiva, las AL no son esquemas sociales inmutables en la medida en que el estatus de una variedad puede cambiar de acuerdo con diversas circunstancias sociales. El juicio social de una variedad estándar o no estándar se relaciona entonces con la teoría de la acomodación comunicativa, a partir de la cual el hablante selecciona un repertorio lingüístico, tomando en cuenta aspectos internos (como son los estados emocionales y psicológicos) y aspectos externos, como el tipo de conversación, las características físicas del canal, la informalidad o formalidad y la dimensión pública 0 privada (Giles \& Powesland, 1975). De este modo, Giles y Ryan (1982) proponen un modelo de AL que posiciona las variaciones de estilo en dos continuums que permiten ubicar diferentes polaridades: el continuum grupo/persona y el continuum estatus (poder)/solidaridad (Moreno, 1998). En este modelo, la publicidad/formalidad y la privacidad/informalidad, en relación con la mayor o menor solidaridad que se desprende de la pertenencia o exterioridad a un grupo social, genera el uso mayor 0 menor de elementos estilísticos que tienen que ver con el estatus de los participantes de la interacción. Este aspecto de la solidaridad lingüística, según Giles y Ryan (1982), es determinante en la supervivencia de las lenguas minoritarias y en que las políticas de planificación de una lengua se lleven a cabo con éxito.

\section{Prestigio, lenguas en contacto y AL}

En las AL que determinan selección de su repertorio lingüístico, el hablante no solamente toma en cuenta aspectos lingüísticos sino también extralingüísticos. Entre estos aspectos se incluye el prestigio, concepto estudiado en los trabajos pioneros de Giles y Powesland (1975) y en el ámbito hispánico por López (1996), Alvar (1996) y Moreno (1990). El concepto de prestigio, según Giles y Powesland (1975) no se relaciona con aspectos intrínsecos de la lengua, sino con las políticas, los procesos de normalización y la normativización. En este sentido, el prestigio está conectado con la órbita social, en cuanto es una selección de los trazos considerados estándar, y con la órbita individual, en cuanto se relaciona con las AL del hablante. El prestigio no cumple solamente un rol en las variedades de una misma lengua sino en las situaciones de lenguas en contacto. En particular, los estudios de Lambert et al. (1960) demuestran que el estatus sociocultural de la respectiva lengua, es decir, su prestigio, se atribuye de acuerdo con sus posibilidades de ascenso social. En este caso, la lengua prestigiosa aparece como dominante y se privilegia su uso en el dominio educativo y público. Del mismo modo, las lenguas que se perciben como menos prestigiosas pueden sufrir desplazamiento lingüístico, como es el caso de la lengua toba en Bolivia (Hecht, 2011) y del quichua en Ecuador (Haboud, 1998). Alvar (1996) por su parte, entiende por prestigio la aceptación de un tipo de conducta considerada 
mejor que otra y cree que es determinante en el momento de establecer la percepción de una variedad lingüística. Justamente esta consideración es lo que confiere el carácter abarcador de la categoría de lengua a una variedad, mientras que confina a dialecto (variedad restringida) a otras variedades. Para Moreno (1998) el prestigio puede ser considerado como actitud o como conducta, "(...) es algo que se tiene y se demuestra, pero también se concede" (p. 187). En otras palabras, es un proceso de "(...) concesión de estima y respeto hacia individuos o grupos que reúnen ciertas características y que lleva a imitación de las conductas de estos individuos o grupos" (p. 187).

De lo anterior es posible inferir que el prestigio no es un fenómeno estático sino un continuum móvil, en el que se atribuyen más o menos valores normativos y estilísticos a determinada variedad de una lengua. Las conceptualizaciones sobre el prestigio de Alvar (1996) y de Moreno (1998) pueden extenderse a las situaciones de lenguas en contacto y, en modo particular, a aquellas en las que un código (lengua mayoritaria) está en situación de ventaja con relación a otro código (lengua minoritaria).

\section{Normas de prestigio}

El estudio realizado en los dialectos del centro de España por Moreno (1990), extrapoló cinco pares conceptuales significativos en el establecimiento de las normas de prestigio: prestigio del individuo / prestigio de la ocupación; prestigio como actitud / prestigio como conducta; prestigio vertical / prestigio horizontal; prestigio sociológico / prestigio lingüístico. Mientras que para Trudgill (1972) el prestigio no siempre se manifiesta de forma expresa, sino que existen ocasiones en las que puede permanecer encubierto (covert prestige). Este último tipo de prestigio es de difícil aproximación puesto que los hablantes mismos pueden no reconocerlo y no siempre responde a criterios normativos. En este sentido, la relación entre AL y prestigio no es unidireccional y transparente en todos los casos. El estudio de las AL debe tener en consideración el rol que desempeña el prestigio entre las diferentes variedades y lenguas objeto de estudio; además estos dos términos comparten una multicausalidad y una multidimensionalidad relacionada con la diversidad de sus componentes (emocionales, relativos al estatus, al estilo, a la relación entre los interlocutores). El prestigio representa un papel en la configuración de los estereotipos y, en consecuencia, pertenece a los componentes cognitivos de las AL (de esta forma incide en el componente conativo). En esta dirección de análisis, estudiar las AL de una comunidad de habla conlleva también un estudio del prestigio de las variedades o lenguas en contacto.

\section{Identidad, lenguas en contacto y AL}

Para Appel y Muysken (1987) existe una relación entre identidad y AL. Esta relación implica que en una sociedad los diferentes grupos sociales o étnicos tienen ciertas actitudes hacia otros grupos, las cuales se transfieren a la lengua del grupo y finalmente, a los hablantes de la lengua de esa comunidad de habla. Según estos 
autores, otros aspectos están conectados con la forma en la que las AL varían de acuerdo con el dominio -público/privado, coloquial/académico, entre otrosrelacionado con la alternancia de códigos en las situaciones de lenguas en contacto. Estas aseveraciones se basan en los hallazgos de Carranza y Ryan (citados en Appel \& Muysken, 1987) sobre grupos de adolescentes chicanos y anglos en Chicago. Los resultados demostraron que el español obtenía una mayor aceptación en el contexto familiar y menor aceptación en el contexto escolar.

El concepto de identidad se relaciona estrechamente con el de grupo, con el de nación y con el de nacionalidad. Para Fishman (1972), las nacionalidades pueden definirse como una unidad sociocultural suprasocial que se opone a otras identidades a través de criterios de autodefinición. Es justamente este carácter amplio, general, el que lo opone al de grupo, que tiene una dimensión más local. Mientras que la nacionalidad y el grupo étnico se distinguen del de nación porque este último implica la existencia de un territorio y una organización política ad boc denominada Estado. Por otra parte, un Estado no necesariamente corresponde a una única nación, pues puede ser plurinacional, y una nación puede estar conformada a su vez por varios grupos étnicos, lo que aparece como una noción más restringida, o por varias nacionalidades, equivalente a una noción más amplia. Con respecto a la relación entre identidad y lengua, Fishman (1999) desde la sociolingüística, propone un modelo de categorización para caracterizar los actos de habla que tienen lugar entre diferentes grupos identitarios que usan lenguas en situación de contacto. Por otra parte, Guibernau y Rex (1997) consideran que no se puede establecer una correspondencia automática entre lengua e identidad, sobre todo en el caso de las identidades que se definen como étnicas, puesto que, pese a que una comunidad se autocaracterice incluso en sentido forte como étnica, no implica que sea usuaria de una lengua diversa a la de otra identidad.

\section{Aprendizaje y formación de AL}

El aprendizaje puede realizarse a partir de la experiencia directa e indirecta; por experiencia directa se entiende la del sujeto en su medio social y por experiencia indirecta, a través de los medios de comunicación y socialización. Uno de los procesos por los cuales se adquieren las AL es la observación del comportamiento ajeno y de las consecuencias de este; así, existe un aprendizaje instrumental a través del cual se prueba directamente si determinada actitud trae consigo ventajas y desventajas.

Con relación al rol de la familia en la formación de AL, Gardner (1973) considera que existen influencias implícitas y explícitas que afectan directamente las actitudes de aceptación o rechazo. Sus observaciones implican que el aprendizaje puede verse afectado de forma activa si los padres estimulan abierta y positivamente la integración a la comunidad lingüística de la lengua meta, o pasiva, o no explícita, si el rechazo se expresa en actitudes involuntarias. Ante la concurrencia de los dos tipos de input, el implícito prima sobre el explícito y condiciona la actitud. 
Por otra parte, Baker (1992) señala que la incidencia de la escuela en situaciones de bilingüismo es preponderante, pues logra formar nuevas actitudes 0 transformar las ya existentes. Esta influencia tiene lugar no solamente a través de la programación didáctica explícita (en otras palabras, de las asignaturas que se incluyen en el currículo), sino también a través del currículo oculto que se relaciona con los valores y creencias que subyacen a la estructura explícita tanto desde el punto de vista de los contenidos disciplinares como de los demás aspectos de la vida escolar.

\section{Enseñanza de lenguas, motivación y AL}

Según Garrett (2010), las AL servirían tanto de input como de output en el proceso de aprendizaje de una lengua extranjera. Son un input, puesto que su favorabilidad potencia el rendimiento de los aprendientes, y son también un output, en cuanto el buen resultado de un curso de lengua genera a su vez actitudes favorables. Por otra parte, las AL inciden en la motivación, aspecto determinante en el grado de competencia comunicativa alcanzada por los aprendientes. Según Gardner y Lambert (1972), existen dos tipos de motivación: instrumental, propia de los sujetos que tienen fines pragmáticos; y la integrativa, relacionada con el deseo de identificarse o llegar a ser miembro de una comunidad de habla. Por otra parte, la existencia de uno u otro tipo de motivación, según Baetens (1982), es definitiva en el mantenimiento o en el desplazamiento de una lengua en situación de contacto. En general, la motivación que se deriva de la integración parece mejorar los resultados del aprendizaje, mientras que la instrumental suele resultar menos eficaz. Según Gardner y Lambert (1972) y Gardner (1973, 2001), la motivación debe redefinirse siguiendo un modelo psicosocial en el que las actitudes del alumno hacia la comunidad de habla de la lengua meta determinen el grado de motivación y este, a su vez, el grado de adquisición de la lengua.

Estas formulaciones llevaron a Gardner (2001) a postular su teoría Integrative Motivation, que concibe la motivación como un ente complejo que contiene los siguientes factores: Integrative o interés auténtico hacia la lengua meta y deseo de integrarse a la comunidad de habla de la misma; Attitudes Toward the Learning Situations, es decir actitudes positivas hacia los componentes de la situación de aprendizaje (docente, pares, proyecto educativo, currículo); Motivation o fuerza que genera la situación de aprendizaje, comprende los recursos empleados para adquirir la lengua meta y también incluye el placer que se experimenta en el aprendizaje. Byram (2004) por su parte formula su Resultative and Motivational Hypothesis, según la cual el éxito en el aprendizaje influye positiva o negativamente en las actitudes hacia la lengua meta, el país y la cultura. Y Brown (2007) considera que las AL tienen una formación temprana en la infancia del aprendiente y que la relación con la motivación es constitutiva en la medida en que la fuerza motivacional del aprendizaje es esencialmente un constructo de actitudes y creencias. Una orientación similar se explicita en los trabajos de Oroujlou y Vahedi (2011), quienes demuestran que las AL integradas a la motivación proveen la fuerza primaria que impulsa el proceso y 
que, a largo plazo, sostienen el esfuerzo requerido para la adquisición de una lengua extranjera.

También existe una relación relevante entre estilos de aprendizaje -campo actual de notable desarrollo en la investigación didáctica- y AL. Tal como señalan Hosseini y Pourmandnia (2013), es indudable que las actitudes y las creencias que los estudiantes poseen en el momento de ingresar a un aula de adquisición de una lengua extranjera hacen parte de su perfil personal de aprendizaje y en este sentido, el estudio de las AL de los aprendientes sería un recurso que debe integrarse a una didáctica que pretenda dar cuenta eficaz de las diferencias en los estilos cognitivos.

Para Malcolm (1987), por su parte, las AL tienen, además de sus componentes individuales, componentes que podrían denominarse pedagógicos, puesto que hacen referencia a la situación educativa (profesores, pares, currículo, etc.). En este sentido, no se pueden considerar las AL presentes en un proceso de aprendizaje como un factor dado y externo, sino como un elemento móvil que puede variar durante la intervención didáctica.

Hallazgos similares, referidos a la forma en que las percepciones acerca del docente inciden en la formación de AL de estudiantes malayos universitarios aprendientes de inglés, se formulan en los trabajos de Ahmed (2015). Para Christ (1997), las AL definen las políticas educativas en el entorno de una institución dada y por lo tanto son las creencias y los estereotipos de los grupos en el ámbito de lo micropolítico los que en última instancia determinan el currículo lingüístico (cuáles lenguas, cuáles estrategias) de una institución.

En lo relacionado con las estrategias de enseñanza incluidas en el currículo, se ha establecido que existe una relación positiva entre Content and Language Integrated Learning (CLIL) y AL. Según Merisuo-Storm (2007), los aprendientes que participan de este tipo de didáctica adquieren unas actitudes más positivas hacia el aprendizaje de las lenguas en general (lo que incide positivamente en sus resultados), en contraste con estudiantes que no se someten a este tipo de didáctica.

Finalmente, en lo que respecta a la relación entre las AL y la enseñanza de lenguas extranjeras, podemos señalar que las primeras se integran a la competencia comunicativa, en la medida en que hacen parte de los aspectos pragmáticos y sociolingüísticos, según la teoría de Hymes (1971). Las AL tienen un papel tanto en la emisión como en la recepción del significado, tal como hemos visto al exponer los aspectos básicos de la teoría de la acomodación comunicativa. Los participantes de una interacción constantemente están procesando información relacionada con el léxico, los turnos de habla, los elementos fáticos, la prosémica y la cinestesia, las normas de cortesía, y lo hacen tomando en cuenta su sistema particular de valores y usos lingüísticos.

\section{Conclusiones}


Si bien los EAL tienen su origen en el terreno de la sociolingüística variacionista, han tenido una notable repercusión en otros campos del conocimiento, tales como la psicología social, la dialectología perceptiva, la etnografía del habla y la lingüística aplicada a la enseñanza de las lenguas. Los valores que los hablantes vinculan a sus percepciones de una lengua tienen repercusiones en la formación de su conciencia lingüística que, a su vez, condiciona los niveles de inseguridad 0 seguridad lingüística.

En cuanto a la definición y caracterización de las AL, claramente hay una diversidad de miradas que han generado una gran heterogeneidad en su aproximación. Actualmente los enfoques se desplazan en dos sentidos constituidos asimismo por binomios opuestos: en el primero, se oponen las caracterizaciones mentalistas (intangibilidad, interioridad) de las AL a las caracterizaciones conductuales (comportamentales, externas), y en el segundo, se oponen las visiones unicomponenciales a las visiones multicomponenciales, en las que entran en juego diferentes aspectos afectivos, cognitivos y comportamentales.

En las situaciones de lenguas en contacto existe una relación estrecha entre identidad de los diferentes grupos sociales y AL con una determinada lengua 0 variedad. En estos casos, las actitudes hacia un grupo social o étnico se convierten en AL hacia su lengua. Los procesos de enseñanza/aprendizaje de una lengua se verían afectados por el ambiente de adquisición de las actitudes lingüísticas (natural 0 formal/institucional). En el plano institucional, las percepciones están determinadas por impresiones afectivas y cognitivas de los pares, el profesorado, la administración y el currículo en sus dimensiones explícitas e implícitas.

Los EAL han sido un punto focal de interés para los estudiosos de los procesos de enseñanza/aprendizaje de lenguas extranjeras y segundas en las últimas décadas, que han dado lugar a un corpus importante de investigaciones que hacen referencia no solo a la forma en que las actitudes hacen parte de la motivación, sino también a la forma en que influencian la didáctica. Derivas futuras de investigación en el campo se orientan a considerar la importancia de las AL en el perfil cognitivo de los aprendientes y en las repercusiones que este aspecto pueda tener en la planeación del currículo, en el diseño de materiales y en las estrategias de evaluación.

\section{Referencias}

Agheyisi, R. \& Fishman, J. (1970). Language Attitudes: A Brief Survey of Methodological Approaches. Anthropological Linguistics, 12(5), 137-157.

Ahmed, S. (2015). Attitudes towards English Language Learning among EFL Learners at UMSKAL. Journal of Education and Practice, 6(18), 6-16.

Alexander, C. \& Norman, Jr. (1967). Attitude as A Scientific Concept. Social Forces, 45, 278-81. https://doi.org/10.2307/2574399 
Allport, G. W. (1935). Attitudes. In C. Murchison (ed.), Handbook of Social Psychology (pp. 798-844). Clark U.P.

Allport, G. W. (1954). The Nature of Prejudice. Addison-Wesley.

Alvar, M. (1996). Dialectología y cuestión de prestigio. En M. Alvar (dir.), Manual de dialectología hispánica. El español de España (pp. 15-21). Ariel.

Appel, R. \& Muysken, P. (1987). Language Contact and Bilingualism. University Press.

Baetens Beardsmore, H. (1982). Bilingualism: Basic Principles. Multilingual Matters.

Baker, C. (1992). Attitudes and Language. Multilingual Matters.

Brown, D. H. (2007). Principles of Language Learning and Teaching. (5th ed.). Longman.

Byram, M. (2004). Routledge Encyclopedia of Language Teaching and Learning. Routledge.

Chein, I. (1948). Behavior Theory and the Behavior of Attitudes: Some Critical Comments. Physcol Rev, 55(3), 175-188. https://doi.org/10.1037/ $\underline{\mathrm{h} 0054019}$

Christ, H. (1997). Language Attitudes and Educational Policy. In R. Wodak \& P. Corson (eds.), Encyclopedia of Language and Education (pp. 1-11). Dordrecht. https://doi.org/10.1007/978-94-011-4538-1_1

Doob, L. W. (1947). The Behavior of Attitudes. Psychological Review, 54, 135-156. https://doi.org/10.1037/h0058371

Fasold, R. (1990). The Sociolinguistics of Language (Volume 2). Blackwell Pub.

Fishbein, M. (1965). A Consideration of Beliefs, Attitudes and Their Relationships. In I.D. Steiner \& M. Fishbein (eds.), Current Studies in Social Psychology (pp. 107-120). Holt, Reinhart and Winston, Inc.

Fishbein, M., \& Ajzen, I. (1975). Belief, Attitude, Intention, and Behavior: An Introduction to Theory and Research. Addison-Wesley.

Fishman, J. (1972). Language and Nationalism: Two Integrative Essays. Newsbury House.

Fishman, J. (1999). Handbook of Language and Ethnic Identity. Oxford University Press.

García, F. (1999). Fundamentos críticos de sociolingüística. Universidad de Almería. 
Gardner, R. C. (1973). Attitudes and Motivation: Their role in Second Language Acquisition. In J. Oller \& J. Ridhards (eds), Focus on the Learner (pp. 235246). Newbury House.

Gardner, R. C. (2001). Integrative Motivation and Second Language Acquisition. In Z. Dörney \& R. Schmidt (eds.), Motivation and Second Language Acquisition (pp. 1-19). Honolulu, Hawaii: University of Hawai'i, Second Language Teaching and Curriculum Center.

Gardner, R. \& Lambert, W. (1972). Attitudes and Motivation in Second Language Learning. Newbury House.

Garrett, P. (2010). Attitudes to Language. Cambridge University Press. https://doi. org/10.1017/CBO9780511844713

Giles, H. (1973). Accent Mobility: A Model and Some Data. Anthropological Linguistics 15(2), 87-105.

Giles, H. (2014). Language Attitudes: Social Determinants and Consequences of Language Variation. In M. Thomas, The Oxford Handbook of Language and Social Psychology (pp. 11-26). Oxford University Press.

Giles, H., \& Powesland, P. F. (1975). Speech Style and Social Evaluation. Academic Press.

Giles, H. \& Ryan, E. B. (1982) Prolegomena for Developing A Social Psychological Theory of Language Attitudes. In E. B. Ryan \& H. Giles (eds.), Attitudes Towards Language Variation: Social and Applied Contexts (pp. 99-115). Edward Arnold.

Green, B. F. (1954). Attitude Measurement in Lindzey. The Handbook of Social Psychology, 1, 335-369.

Guibernau, M. \& Rex, J. (1997). The Ethnicity Reader. Nationalism, Multiculturalism and Migration. Polity Press.

Haboud, M. (1998). Quichua y castellano en los Andes. Los efectos de un contacto prolongado. Ediciones Abya-Yala.

Hecht, A. C. (2011). Encrucijadas entre las políticas educativas y el mantenimiento de las lenguas indígenas de Argentina. Espaço Amerindio, 4, 92-116. https://doi. org/10.22456/1982-6524.11865

Hosseini, S. B., \& Pourmandnia, D. (2013). Language Learners' Attitudes and Beliefs: Brief Review of the Related Literature and Frameworks. International Journal on New Trends in Education and Their Implications, 4(4), 63-74. 
Hymes, D. (1971). Competence and Performance in Linguistic Theory. In R. Huxley \& E. Ingram (eds.), Language Acquisition: Models and Methods (pp. 67-99). Academic Press.

Hudson, R. A. (1980). Sociolinguistics. Cambridge University Press.

Labov, W. (1972). Sociolinguistic Patterns. University of Pennsylvania Press.

Lambert, W. (1967). A Social Psychology of Bilingualism. JSI, 13(2), 91-109. https:// doi.org/10.1111/j.1540-4560.1967.tb00578.x

Lambert, W. W. \& Lambert, W. E. (1964). Chapter 4. In W. Lambert \& W. E. Lambert (eds.), Social Psychology (pp. 49-69). Englewood Cliffs.

Lambert, W. et al. (1960). Evaluational Reactions to Spoken Languages. Journal of Abnormal and Social Psychology, 60, 44-51. https://doi.org/10.1037/ $\underline{\mathrm{h} 0044430}$

La Piere, R. T. (1934). Attitudes vs. Action. Social Forces, 13, 230-237. https://doi. org/10.2307/2570339

López, J.M. (1996). La norma lingüística hispánica "Los hispanohablantes y la unidad de la lengua española”. En Z. Estrada, M. Figueroa \& G. López (eds.), Memorias del III Encuentro de Lingüística del Noroeste (pp. 11-28). Universidad de Sonora.

López, H. (1989). Sociolingüística. Gredos.. https://doi.org/10.1515/ijsl.1989.77.55

Malcolm, I.G. (1987). Continuities in Communicative Patterns in Crossculturals Classrooms. In B.K. Das (ed.), Communication and Learning in The Classroom Community (pp. 37-63). Singapore University Press.

Merisuo-Storm, T. (2007). Pupils' Attitudes Towards Foreign-Language Learning and The Development of Literacy Skills in Bilingual Education. Teaching and Teacher Education, 23(2), 226-235.. https://doi.org/10.1016/j.tate.2006.04.024

Miquel, L. (2004). La subcompetencia sociocultural. En J. Sánchez Lobato \& S. Gargallo, (dirs.), Vademécum para la formación de profesores. Enseñar español como segunda lengua (L2)/Lengua extranjera (LE) (pp. 511-532). SGEL.

Moreno, F. (1990). Metodología sociolingüística. Gredos.

Moreno, F. (1998). Principios de sociolingüística y sociología del lenguaje. Ariel.

Oppenheim, B. (1982). An Exercise in Attitude Measurement. In G. Breakwell (ed.), Social Psychology: A Practical Manual (pp. 38-56). Macmillan Education. https://doi.org/10.1007/978-1-349-16794-4_3 
Oroujlou, N. \& Vahedi, M. (2011). Motivation, Attitude, and Language Learning. Procedia-Social and Behavioral Sciences, 29, 994-1000. http://dx.doi. org/10.1016/j.sbspro.2011.11.333

Osgood, C. et al. (1957). The Measurement of Meaning. University of Illinois Press.

Rokeach, M. (1968). The Nature of Attitudes, International Encyclopedia of Social Sciences. Macmillan.

Rosenberg, M. J. (1960). A Structural Theory of Attitude Dynamics. Public Opinion Quarterly, 24, 319-40. https://doi.org/10.1086/266951

Schneiderman, E. (1976). An Examination of the Ethnic and Linguistic Attitudes of Bilingual Children. RAL, 33, 59-72. https://doi.org/10.1075/itl.33.04sch

Street Jr, R. L. \& Hopper, R. (1982). A Model of Speech Style Evaluation. In E. Bouchard Ryan \& H. Giles (eds.), Attitudes Toward Language Variation: Social and Applied Contexts (175-188). Edward Arnold.

Trudgill, P. (1972). Sex, Covert Prestige and Linguistic Change in the Urban British English of Norwich. Language in Society, 1(2), 179-195. https://doi. org/10.1017/S0047404500000488

White, S. (1989). Backchannels Across Cultures: A Study of Americans and Japanese. Language in Society, 18(1), 59-76. https://doi.org/10.1017/ $\underline{\mathrm{S} 0047404500013270}$ 